5. Hentrich M, Schalk E, Schmidt-Hieber M, et al. Central venous catheter-related infections in hematology and oncology: 2012 updated guidelines on diagnosis, management and prevention by the Infectious Diseases Working Party of the German Society of Hematology and Medical Oncology. Ann Oncol 2014;25:936-947.

6. Pepin CS, Thom KA, Sorkin JD, et al. Risk factors for central line-associated bloodstream infections: a focus on comorbid conditions. Infect Control Hosp Epidemiol 2015;36:479-481.

7. Schalk E, Färber J, Fischer T, Heidel FH. Central venous catheterrelated bloodstream infections in obese hematologic patients. Infect Control Hosp Epidemiol 2015;36:995-996.

\section{Low Incidence of Central Venous Catheter- Related Bloodstream Infections in Stem Cell Transplant Patients in Eastern India Despite High Community Burden of Multidrug-Resistant Pathogens}

To the Editor-Central venous catheters (CVCs) are indispensable in hematopoietic stem cell transplant practice. With the increasing burden of multidrug-resistant bacterial organisms (MDROs) in communities worldwide, the risk of CVC colonization and subsequent life-threatening bloodstream infections (BSI) with these organisms poses a serious threat to transplant practice. ${ }^{1,2}$ Hence, safety management of central lines presents major challenges in the setting of hematological disorders. We evaluated our experience with CVC MDRO colonization and bacteremia at the stem cell transplant (SCT) unit in the Tata Medical Center, Kolkata, India.

A retrospective cohort analysis was conducted from December 2011 to June 2015. Patients were nursed in single en-suite high-efficiency particulate air (HEPA)-filtered rooms. The air quality of each bone-marrow-transplant room was maintained using a separate air handling unit; air quality was checked using a hand-held air particle counter; air velocity was measured using an anemometer, and air pressure was monitored using a differential pressure monitor. ${ }^{3}$ Water quality in the SCT rooms was maintained using Pall filters for shower heads and taps. These filters have a $0.2-\mu \mathrm{m}$ pore size and provide a protective barrier against waterborne contaminants including Legionella spp., and Pseudomonas spp., and other bacteria (coliforms). The water quality of the hospital is maintained through reverse osmosis and chlorination (free chlorine, $0.2-0.5 \mathrm{ppm})^{4}$

The patient:nurse ratio in the SCT unit was 1:1.5. Nurses in the SCT unit were trained using the World Health Organization (WHO) module of hand hygiene, preparation and administration of medicines (drugs, blood products) under aseptic techniques, and CVC dressing protocol, as well as standard precautions. Housekeeping (environmental cleaning) practices included a 3 -shift cleaning protocol using soap and water and sodium hypochlorite (for floors); 10\% hydrogen peroxide (for all surfaces pre-admission and post discharge); and alcohol (isopropyl alcohol and ethyl alcohol for beds, steel surfaces, and biomedical equipment). Before insertion of a CVC, each patient was given a bath with $4 \%$ chlorhexidine in addition to soap and water. The CVC care bundle included sterile insertion under maximum barrier precautions, daily change of infusion sets, gauze or transparent dressing, flushing and locking, as well as the use of an ELD96 filter (Pall) for total parenteral nutrition and a Lipipor filter (Pall) for parenteral lipids. We also used a leucodepletion filter for packed cells and platelets. A dose of antibiotic (co-amoxiclav) was given pre-operatively within 60 minutes of CVC insertion. CVCs were inserted a few days prior to transplant, and where feasible, most CVCs were removed within 90 days of insertion. Skin preparation before insertion was done using 2.5\% chlorhexidine with isopropyl alcohol 70\% (or $10 \%$ povidone iodine). Patients and family members were educated to take care of the central line at home and to report to the outpatient department for heparinized flushing, blood sampling, and dressing weekly. Before accessing the line, meticulous hub scrubbing was done for 20-30 seconds with $2.5 \%$ chlorhexidine and $70 \%$ isopropyl alcohol. The line sites were evaluated on every shift for signs of inflammation and infection of the exit site. Bed linens for each the patient were sterilized by autoclaving and changed daily. Patients were provided with a "neutropenic diet" (pressure cooked food and water). Stem cells were collected, stored, and infused under aseptic techniques. All cases of clinical sepsis and positive blood cultures were proactively treated using a protocol of empirical therapy (piperacillin-tazobactam + amikacin $>$ meropenem + teicoplanin $>$ meropenem + colistin), and positive blood culture directed early therapy based on Gram-stain findings and preliminary sensitivity. ${ }^{5}$

During the study period, a total of 170 CVCs were placed in 163 patients to support 172 transplants, including 112 allogeneic procedures. Nearly one-third of allogeneic transplants (36 of 112) were high risk, including HLA haplotype-matched procedures (28 of 112) and matched-unrelated donor procedures (8 of 112). The median age of patients was 32 years (range, 2-66 years), 123 (75\%) were male. The majority of CVCs were tunneled devices (Hickman catheters; 113 of 170); the rest were peripherally inserted central lines (PICCs; 10 of 170 ) and non-tunneled central lines (47 of 170) inserted into jugular or subclavian veins. Pre-transplant surveillance cultures of stool and throat swabs identified MDROs in 145 (87\%) and $42(26 \%)$ patients, respectively. ${ }^{6}$ Stool surveillance showed ESBL in 87 patients $(60 \%)$, carbapenemase producers in 43 patients $(30 \%)$, AmpC producers in 26 patients (18\%), and VRE in 4 patients $(2.7 \%)$. Throat surveillance showed carbapenemase producers in 23 patients tested (55\%), ESBL in 17 patients (40\%), AmpC producers in 4 patients $(9.5 \%)$, and VRE in 1 patient $(2.3 \%)$ (Table 1$)$.

The median duration of neutropenia $\left(\leq 500 / \mathrm{mm}^{3}\right)$ was 11 days (3-36 days). A cumulative 11,410 CVC days were recorded, with a median 58 days per patient (range, 8-418 days, interquartile range, $18-89$ days). A total of 36 BSIs were observed, including 9 isolates identified in pre-transplant 
тавLE 1. Distribution of Multidrug-Resistant Organisms (MDROs) in Surveillance Cultures

\begin{tabular}{|c|c|c|c|c|c|}
\hline & $\begin{array}{l}\text { Total No. of } \\
\text { Patients }\end{array}$ & $\begin{array}{l}\text { ESBL, No. } \\
\quad(\%)\end{array}$ & $\begin{array}{c}\text { AmpC, No. } \\
(\%)\end{array}$ & $\begin{array}{c}\text { Carbapenemase Producer, No. } \\
(\%)\end{array}$ & $\begin{array}{l}\text { VRE, No. } \\
\quad(\%)\end{array}$ \\
\hline Stool surveillance culture for MDRO & 145 & $87(60)$ & $26(18)$ & $43(30)$ & $4(2.7)$ \\
\hline
\end{tabular}

NOTE. ESBL, extended-spectrum $\beta$-lactamase; AmpC, a type of $\beta$-lactamase; VRE, vancomycin- resistant enterococcus.

cultures ( 7 from stool, 2 from throat swabs). A total of 19 BSIs were associated with mucosal barrier injury (MBI-LCBI: mucosal barrier injury-laboratory-confirmed bloodstream infection). ${ }^{7}$ Thus, 17 BSIs were associated with central-line infection, with a CLABSI rate of 1.48 per 1,000 catheter days. In addition, $7(4 \%)$ lines were removed for various reasons (eg, exit-site infection, tip migration, fracture of the line). No CVC was removed because of CLABSI.

Despite a high rate of MDRO colonization, a low incidence of catheter-associated bacteremia was observed in patients undergoing stem cell transplant at our center. This finding suggests that in high-intensity clinical settings, despite the high prevalence of MDROs, a combination of rigorous central venous catheter care and concerted infection vigilance ensures low catheter-associated bloodstream infection rates. Coordinated team work among various multi-disciplinary teams (ie, maintenance engineering, nursing, housekeeping, infection control, SCT unit doctors and central sterile supply department, transfusion medicine department, patients and relatives) led the way to attaining a satisfactory outcome in this challenging setting.

\section{ACKNOWLEDGMENTS}

Financial support: No financial support was provided relevant to this article. Potential conflicts of interest: All authors report no conflicts of interest relevant to this article.

Mita Roychowdhury, BSc, MSc;
Jeevan Kumar, MBBS, MD, DNB;
Anupam Chakrapani, MD, DM;
Saurabh Jayant Bhave, MBBS, MD;
${ }^{2}$
Shekhar Krishnan, MBBS, MRCP, FRCPath, PhD; ${ }^{3}$
Robin Thambudorai, MBBS, MS; ${ }^{4}$
Bhattacharya, MD, DNB, DipRCPath, FRCPath;
Mammen Chandy, MD, FRCPA, FRACP, FRCP

Affiliations: 1. Department of Nursing, Tata Medical Center, Kolkata, India; 2. Department of Clinical Hematology, Tata Medical Center, Kolkata, India; 3. Department of Pediatric Oncology, Tata Medical Center, Kolkata, India; 4. Department of Gastro-Intestinal and Hepatobiliary Surgery, Tata Medical Center, Kolkata, India; 5. Department of Microbiology, Tata Medical Center, Kolkata, India

Address correspondence to Dr. Sanjay Bhattacharya, MD, DNB, DipRCPath, FRCPath, Consultant Microbiologist, Tata Medical Center, 14 Major Arterial Road (E-W), New Town, Kolkata 700 156, India (drsanjay1970@hotmail.com). Infect Control Hosp Epidemiol 2016;37:619-620

(C) 2016 by The Society for Healthcare Epidemiology of America. All rights reserved. 0899-823X/2016/3705-0024. DOI: 10.1017/ice.2016.18

\section{REFERENCES}

1. Bhattacharya S, Goel G, Mukherjee S, Bhaumik J, Chandy M. Epidemiology of antimicrobial resistance in an oncology center in eastern India. Infect Control Hosp Epidemiol 2015;36: 864-866.

2. Bhattacharya S, Das D, Bhalchandra R, Goel G. Patient isolation in the high-prevalence setting: challenges with regard to multidrug-resistant Gram-negative bacilli. Infect Control Hosp Epidemiol 2013;34:650-651.

3. Bhalchandra R, Bhattacharya S, Ratnam Soundaranayagam J, Garai S, Chandy M. Importance of air particle counts in hospital infection control: insights from a cancer center in eastern India. Infect Control Hosp Epidemiol 2015;36:1115-1117.

4. Bhalchandra R, Chandy M, Ramanan VR, Mahajan A, Soundaranayagam JR, Garai S, Bhattacharya S. Role of water quality assessments in hospital infection control: experience from a new oncology center in eastern India. Indian J Pathol Microbiol 2014;57:435-438.

5. Goel G, Das D, Mukherjee S, Bose S, Das K, Mahato R, Bhattacharya S. A method for early detection of antibiotic resistance in positive blood cultures: experience from an oncology centre in eastern India. Indian J Med Microbiol 2015; 33(Suppl):53-58.

6. Landman D, Salvani JK, Bratu S, Quale J. Evaluation of techniques for detection of carbapenem-resistant Klebsiella pneumoniae in stool surveillance cultures. J Clin Microbiol 2005;43:5639-5641.

7. Metzger KE, Rucker Y, Callaghan M, Churchill M, Jovanovic BD, Zembower TR, Bolon MK. The burden of mucosal barrier injury laboratory-confirmed bloodstream infection among hematology, oncology, and stem cell transplant patients. Infect Control Hosp Epidemiol 2015;36:119-124.

\section{Lost in Transition: Discontinuity of Care During Patient Transfer}

To the Editor-On my weekly device rounds at a longterm acute care hospital (LTACH), I asked my patient 5 days into his stay for treatment of osteomyelitis: "Sir, why do you have this urinary catheter?" He shrugged his shoulders while walking in the corridor and said, "I don't know. They put it in about 10 days ago while I was in the hospital." 